\title{
Mucosal immunity to mycobacteria in leprosy patients and their contacts
}

\author{
I A CREE, SUSAN SHARPE, N D C STURROCK, \\ I H COCHRANE*, W C SMITH \& J SWANSON BECK \\ Departments of Pathology and Community Medicine, University of \\ Dundee, Ninewells Hospital and Medical School, Dundee, DD1 \\ 9SY, Scotland; *HEED Leprosy Hospital, Kamalganj, PO Kera- \\ matnagar, District Moulvibazar, Bangladesh
}

Accepted for publication 24 June 1988

\begin{abstract}
Summary Since the development of leprosy may follow the formation of a n initial lesion in the nose, mucosal immune responses might be important in the protective immune response to Mycobacterium leprae. Salivary antibody responses to $M$. leprae and other mycobacteria were therefore investigated in leprosy patients and healthy contacts using ELISAs against whole mycobacteria and an $M$. leprae-specific glycolipid constituent (PGL1) of the external surface of $M$. leprae. Lower levels of salivary $\operatorname{IgA}$ directed against $M$. leprae were found in household contacts (at high risk of developing leprosy) than in hospital contacts (low risk of leprosy). Samples from the local indigenous population with no known leprosy contact showed an intermediate number of positive salivary $\operatorname{IgA}$ responses against $M$. leprae and untreated patients were less likely to be positive than treated patients.

Correlation was found between salivary antibody responses to $M$. leprae, $M$. scrofulaceum and $M$. tuberculosis, suggesting the presence of some crossreacting antibody. Few patients and no healthy subjects had detectable antibody responses against an epitope of PGLl, suggesting that this important serum antibody response is not a major component of the mucosal immune response to $M$. leprae. Since there appears to be a secretory $\operatorname{IgA}$ response to $M$. leprae which is least likely to be found amongst those with the disease and in those individuals with increased risk of developing leprosy, we suggest that the mucosal immune system might be of importance in a putative protective response to infection by the leprosy bacillus.
\end{abstract}

\section{Introduction}

Leprosy bacilli are excreted in large numbers from the nose in lepromatous leprosy ${ }^{1}$ and it is widely believed that the nose may also be the site of initial infection, although other methods of transmission cannot be excluded at present. ${ }^{2}$ By analogy with tuberculosis, it seems feasible that local cell-mediated immunity (CMI) might be important in producing resolution of the initial 
infection. ${ }^{3}$ However, organisms entering the upper respiratory tract must penetrate epithelial barriers before establishing an infection and so mucosal immunity appears to offer an alternative explanation for the successful def ence against Mycobacterium leprae mounted by the vast majority of people coming into contact with the disease. ${ }^{4}$ This might involve interference with the adherence of inhaled bacilli to mucosal surfaces. Although the mechanism(s) involved in adherence of $M$. leprae to cells are not fully understood, adherence of other bacteria to mucosal surfaces may involve specific receptors, ${ }^{5}$ or may depend upon the hydrophobicity of the organism..${ }^{67}$ Prevention of adherence depends upon physical factors such as a constant flow of mucus which traps organisms and on the secretion of humoral factors such as lysozyme and immunoglobulins (predominantly $\operatorname{IgA}$ ) directed against bacterial antigens. ${ }^{8,9}$

Until recently, the few published studies of mucosal immunity in leprosy have concentrated on patients $\mathrm{s}^{10,11}$ who by getting the disease have demonstrably failed immunologically. Abe et al. ${ }^{11}$ showed that most lepromatous patients do not produce a salivary IgA response to $M$. leprae and that treated patients are more likely to show a response than active cases. The same authors ${ }^{12}$ have recently shown that a high proportion of contacts of leprosy patients have detectable IgA against $M$. leprae in the saliva, which they hope might prove useful for the early diagnosis of leprosy.

Salivary IgA responses to $M$. leprae might well be linked to mucosal and systemic CMI responses, since both may result from mucosal presentation of antigen. ${ }^{8,13}$ Healthy contacts of untreated leprosy patients are the most likely subjects to be challenged continuously by $M$. leprae in an endemic population and a high proportion of these individuals are likely to be producing a protective immune response. In this investigation we sought salivary antibodies to mycobacteria in two contact groups: household contacts of untreated leprosy patients (who have an increased risk of leprosy), and hospital workers who are also exposed daily to live leprosy bacilli, but who have a low risk of developing leprosy. For comparison, we have included groups of untreated patients, treated patients and unselected subjects from the indigenous population in the study.

The ELISA methods were chosen because they detected antibody to surface antigens on $M$. leprae and other species of mycobacteria. Salivary IgA antibody cross-reacting with other mycobacteria might also be effective in preventing adherence of $M$. leprae, and consequently the lack of strict immunological specificity of the whole cell mycobacterial ELISA used was considered advantageous. Since the saliva samples for the first study in Bangladesh were chemically preserved for transport back to Scotland for analysis (a procedure which might have altered the levels of $\operatorname{IgA}$ present), a second study was performed in Fiji using an ELISA developed for use in the field. This also allowed comparison not only of the methodological factor but also of the salivary antibody responses of two geographically distinct populations in which leprosy is endemic.

\section{Materials and methods}

\section{SUBJECTS}

The saliva samples used for the initial study were obtained by IAC during a field study in northern Bangladesh in $1986 .{ }^{14} \mathrm{~A}$ total of 253 subjects were included in the investigation. These were divided into five groups: 52 untreated leprosy patients, 53 treated leprosy patients, 78 household contacts of untreated patients, 20 hospital contacts, and 50 indigenous control subjects. The Ridley-Jopling classification ${ }^{15,16}$ was confirmed by skin biopsy in 41 of the untreated patients and in 11 of the treated patients. The age and sex distribution in each group is given in Table 1. The untreated patients were classified on the Ridley-Jopling scale as 4 TT, 23 BT,1 BB, 9 BL, 8 LL and 7 Idt, while there were $29 \mathrm{BT}, 1 \mathrm{BB}, 9 \mathrm{BL}, 14 \mathrm{LL}$ patients in the treated group.

Following this study, one of the ELISA methods used was adapted for use in the field and a further survey was performed amongst leprosy patients and their contacts in Fiji. A total of 163 subjects were examined, including 56 treated leprosy patients, 24 household contacts of treated patients, 23 hospital workers, 39 indigenous subjects (mainly medical students), and 21 treated 
Table 1. The age and sex distribution of Bangladeshi and Fijian subjects

\begin{tabular}{|c|c|c|c|c|c|}
\hline \multirow[b]{2}{*}{ Group } & \multirow[b]{2}{*}{ No. } & \multirow{2}{*}{$\begin{array}{l}\text { Male: female } \\
\text { ratio }\end{array}$} & \multicolumn{2}{|c|}{ Age (years) } & \multirow[b]{2}{*}{$\%<15$ years } \\
\hline & & & Mean & SD & \\
\hline \multicolumn{6}{|l|}{ Bangladeshi series } \\
\hline New patients & 52 & $1: 0 \cdot 86$ & $33 \cdot 1$ & $11 \cdot 9$ & $5 \cdot 8$ \\
\hline Treated patients & 53 & $1: 0 \cdot 20$ & $34 \cdot 1$ & $10 \cdot 5$ & 0 \\
\hline Household contacts & 78 & $1: 0 \cdot 95$ & $23 \cdot 4$ & $15 \cdot 0$ & $35 \cdot 9$ \\
\hline Hospital contacts & 20 & $1: 0 \cdot 82$ & $35 \cdot 2$ & $11 \cdot 1$ & 0 \\
\hline Control group & 50 & $1: 0 \cdot 79$ & $24 \cdot 9$ & $8 \cdot 1$ & 0 \\
\hline Total & 253 & $1: 0 \cdot 68$ & $28 \cdot 9$ & $12 \cdot 9$ & $12 \cdot 2$ \\
\hline \multicolumn{6}{|l|}{ Fiji series } \\
\hline Treated patients & 56 & $1: 0 \cdot 72$ & $49 \cdot 6$ & $19 \cdot 8$ & $3 \cdot 6$ \\
\hline Hospital contacts & 23 & $1: 1 \cdot 88$ & $39 \cdot 7$ & $10 \cdot 9$ & 0 \\
\hline Household contacts & 24 & $1: 1 \cdot 67$ & $26 \cdot 8$ & $13 \cdot 2$ & $16 \cdot 7$ \\
\hline Indigenous population & 39 & $1: 1 \cdot 86$ & $22 \cdot 7$ & 9.9 & 0 \\
\hline Treated TB patients & 21 & $1: 0 \cdot 91$ & $31 \cdot 2$ & $14 \cdot 4$ & $4 \cdot 8$ \\
\hline Total & 163 & $1: 0 \cdot 90$ & $35 \cdot 9$ & $18 \cdot 7$ & $4 \cdot 3$ \\
\hline
\end{tabular}

tuberculosis patients. The age and sex distribution of the subjects is given in Table 1. The treated patient group were classified clinically on the Ridley-Jopling scale as 9 TT, 5 BT, 2 BB, 14 BL, and $26 \mathrm{LL}$.

\section{SALIVA COLLECTION AND PRESERVATION}

Saliva was collected by asking subjects to suck orange-flavoured multivitamin tablets (Haliborange, Evans Medical, Beaconsfield, England) and expel saliva over a period of 5 minutes. Large particles of food were removed and the saliva samples were preserved for transport by the addition of the following chemicals (final concentrations in parentheses) to inhibit bacterial growth and enzymatic proteolysis: sodium azide $(0 \cdot 5 \mathrm{~g} / \mathrm{l})$, iodoacetamide $(2.00 \mathrm{mM})$, phenylmethylsulphonylfluoride $(0 \cdot 34$ $\mathrm{mM})$, and ethylenediamine tetra-acetic acid $(2.00 \mathrm{mM})$. Prior to the field project, measurement of total IgA levels by nephelometry in saliva samples kept at room temperature for 3 months showed no appreciable loss of IgA. Following transport to Scotland, the saliva samples were centrifuged at $11,000 \mathrm{G}$ for $15 \mathrm{~min}$ in a MSE Microcentaur centrifuge and divided into 3 equal aliquots, 2 of which were frozen for future study. Three of the saliva samples were lost in transit. In the Fijian study, vitamin C tablets (Vit Valu Laboratories, Sterling Pharmaceuticals Ltd, Sydney, Australia) were used to stimulate salivation and the samples were ref rigerated until the ELISA could be performed (maximum delay: $12 \mathrm{hr}$ ).

\section{ELISAS}

ELISA studies using whole y-irradiated mycobacteria ( $M$. leprae, $M$. tuberculosis and $M$. scrofulaceum) provided by Dr R J W Rees (Division of Communicable Diseases, Clinical Research Centre, Harrow) were perf ormed on all of the saliva samples. The saliva samples from Bangladesh were screened for IgA, IgM and IgG against $M$. leprae, and for antibody of any subclass against whole y-irradiated $M$. scrofulaceum and $M$. tuberculosis (strain H37Rv). In addition, the Bangladeshi samples were screened in an ELISA using a BSA glycoconjugate (also provided by Dr R J W Rees) which mimics a major epitope of the phenolic glycolipid (PGL1) of M. leprae. ${ }^{17}$ This glycoconjugate consists of the intact terminal disaccharide of the natural antigen coupled to BSA by reductive amination and corresponds to conjugate 11 as used by Brett et al. ${ }^{17}$ The Fijian samples were screened for anti- $M$. leprae IgA only. 
For the ELISA using whole mycobacteria, plastic ELISA plates (Nunclon, Gibco Ltd, Paisley, Scotland) were treated with $0 \cdot 1 \%$ gelatine and dried at $60^{\circ} \mathrm{C}$ for $3 \mathrm{~h}$ before adding whole mycobacteria to the wells at a concentration of $10^{7} / \mathrm{ml}$ in a volatile ammonium carbonate buffer $(\mathrm{pH}$ $8 \cdot 2$ ) and drying overnight at $37^{\circ} \mathrm{C}$. The wells were blocked with $0 \cdot 1 \%$ bovine serum albumin (BSA) in phosphate buffered saline ( $\mathrm{pH} 7 \cdot 1$ ) (PBS) for $1 \mathrm{~h}$ at $37^{\circ} \mathrm{C}$, and washed 4 times over 15 min with $0.1 \%$ BSA in PBS before the saliva samples were added. Saliva samples from Bangladesh were diluted $1: 10$ in $0.1 \%$ BSA in PBS and incubated in prepared plates at $37^{\circ} \mathrm{C}$ for $90 \mathrm{~min}$. The plates were washed 4 times bef ore horseradish peroxidase (HRP) conjugated antihuman immunoglobulin (against IgA, IgM, IgG or polyvalent) was added (1:1000 dilution) and incubated at $37^{\circ} \mathrm{C}$ for 90 min. Following a further 4 washes, the HRP was developed by incubation for $30 \mathrm{~min}$ at $37^{\circ} \mathrm{C}$ with freshly prepared $0.4 \mathrm{mg} / \mathrm{ml}$ o-phenylenediamine (OPD) containing $0.05 \%$ hydrogen peroxide in a citrate-phosphate buffer ( $\mathrm{pH} 5 \cdot 0)$. The reaction was stopped with $2.5 \mathrm{~N}$ sulphuric acid and the results read at $490 \mathrm{~nm}$ using an automatic Dynatech MR 580 microELISA reader.

For use in Fiji this technique was modified by: 1, using undiluted saliva; and 2, by the use of 2 $\mathrm{mM} 2,2^{\prime}$-azino bis(3-ethylbenzthiazoline sulphonic acid) (ABTS) with $5 \mathrm{mM}$ hydrogen peroxidase as the final substrate. ${ }^{18}$ All incubations following coating of the bacilli onto the wells were performed at $24^{\circ} \mathrm{C}$, necessitating a longer incubation time for the saliva $(2.5 \mathrm{~h})$. The HRP-ABTS reaction was stopped with $0 \cdot 1 \%$ sodium dodecyl sulphate (SDS) and read visually against a white background as positive or negative by comparing wells with $M$. leprae and wells without $M$. leprae. Since the ABTS reaction was being used under field conditions for the first time, the opportunity was taken to compare the effect of ABTS incubation at $24^{\circ} \mathrm{C}$ and $32^{\circ} \mathrm{C}$. The results differed in 15 out of the 163 cases, in all of which the $24^{\circ} \mathrm{C}$ assay was positive and the $32^{\circ} \mathrm{C}$ assay negative.

\section{DATA ANALYSIS}

The results of the ELISAs are expressed as the difference in absorbance between the mean of triplicate wells coated with the whole mycobacteria or the glycoconjugate and a single well without mycobacteria. Since background absorbances in both the whole mycobacterial and BSA glycoconjugate ELISAs varied between 0 and 0.030 , an insignificant level in most positive specimens, a single control well was used. This allowed 3 wells to be coated with antigen to reduce errors between positive salivas (maximum coefficient of variation $=20 \%$ ) and as a result it was possible for all of the saliva samples to be screened at one time.

Since the whole mycobacterial assay does not distinguish between specific and cross-reacting antibody, no unequivocally negative control group could be tested to determine a cut-off point for the Bangladeshi series. Theref ore the absorbance differences were compared directly for each group using nonparametric statistical methods.

The results from the Fijian study were subjectively determined as positive or negative, and it theref ore was necessary to define an arbitrary cut-off point for comparison of the Bangladeshi data with the Fijian results. Since $82 \%$ of the Fijian treated patient group were positive and this group was the only one common to both studies, the cut-off point was defined as the absorbance value at which $82 \%$ of the Bangladeshi treated patients would be regarded as positive.

The results were analysed using Statgraphics (STSC, California, USA) software. The results of the Bangladeshi assays were compared using Spearman Rank Correlation and the Mann-Whitney U test.

\section{Results}

Salivary $\operatorname{IgA}$ against whole $M$. leprae was detectable in some individuals in all of the Bangladeshi subject groups (Figure 1). Occasional saliva samples produced high background values in wells coated with gelatine, but not $M$. leprae. These patients may have antibody to gelatine in their saliva and were therefore excluded from statistical analysis. In the leprosy patients, comparison of the 


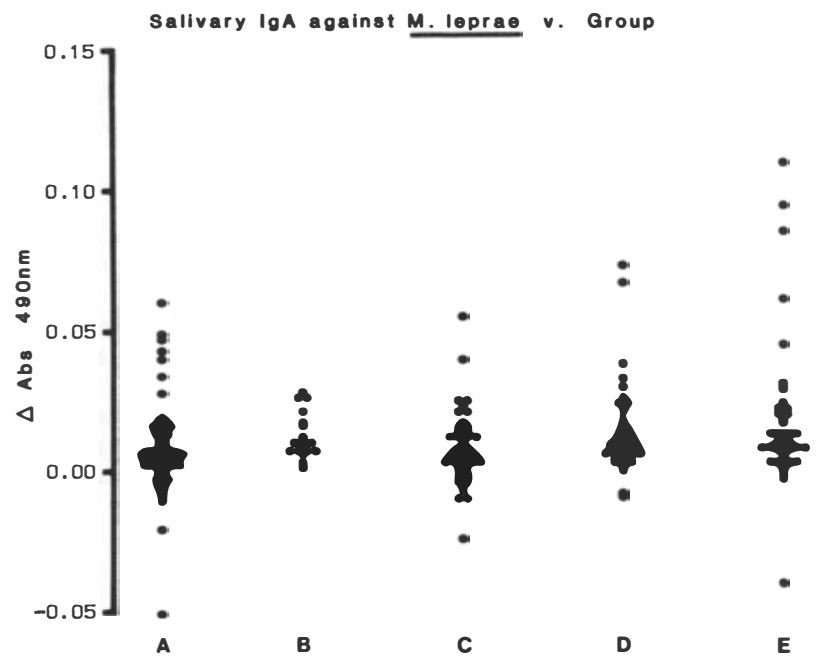

Figure 1. Salivary IgA antibody response to whole y-irradiated $M$. leprae for each subject group. A, household contacts; B, hospital contacts; C, untreated patients; D, treated patients; and E, indigenous subjects.

salivary IgA titres to $M$. leprae with the Ridley-Jopling classification showed no discernible pattern of response. The ELISA for IgM anti- $M$. leprae antibodies showed lower absorbance differences than the IgA ELISA, but the general distribution of the results was a similar pattern in both Ig subclasses and there is weak rank correlation between the results of these ELISAs for all subjects $(r=0 \cdot 29, P<0 \cdot 001)$. This relationship appears to derive from a stronger correlation between the $\operatorname{IgA}$ and $\operatorname{IgM}$ results in the treated patient group $(r=0.55, P<0.001)$ and in the indigenous control group $(r=0.49, P<0 \cdot 001)$. The ELISA for salivary IgG against $M$. leprae was uniformly negative in all subject groups.

The percentage of positive IgA responses against $M$. leprae in each subject group from both Bangladesh and Fiji are shown in Table 2. The low percentage of positive results amongst the household contacts compared with the other groups is particularly striking, and is apparent in both study populations. When the results from the study groups in Bangladesh (Figure 1) are compared using the Mann-Whitney U Test, significantly lower absorbance differences are obtained in the household contacts than hospital contacts $(P<0 \cdot 01)$, although both these groups have daily exposure to $M$. leprae. The household contacts also have a significantly lower IgA anti-M. leprae response than the treated patient group $(P<0.005)$ and the indigenous subjects $(P<0.003)$. The larger proportion of positive responses amongst treated patients compared with untreated patients is also statistically significant $(P<0 \cdot 05)$.

The results of the ELISAs for polyvalent antibody against $M$. tuberculosis and $M$. scrofulaceum (Figure 2(a) and (b), show significant correlation with the IgA response to $M$. leprae $(r=0 \cdot 29$, $P<0.001$ and $r=0.21, P<0.001$ respectively) and with each other $(r=0.33, P<0.001)$. This suggests that there is a salivary antibody response to closely related epitopes on external surface antigens of $M$. leprae and the other mycobacteria studied. However, the results from treated tuberculosis patients in Fiji shows that these subjects have similar positivity for salivary IgA against $M$. leprae as the indigenous healthy population. If the whole $M$. leprae ELISA was detecting antibody against $M$. tuberculosis as well as $M$. leprae, one might expect a considerably higher number of positive responses in tuberculosis patients. Nevertheless, the rates of positivity in each of the subject groups from Bangladesh in the ELISA for antibody against whole $M$. tuberculosis are similar to those seen in the IgA anti- $M$. leprae assay. The household contact group have lower responses in the $M$. tuberculosis ELISA than the hospital contacts $(P<0 \cdot 001)$, the treated patients $(P<0.001)$, and the indigenous subjects $(P<0.001)$. There is also a lower mucosal antibody 
Table 2. Percentage of positive responders (numbers in brackets) in ELISA for salivary $\operatorname{IgA}$ against $M$. leprae for Bangladesh and Fiji populations. Cut-off value for Bangladesh ELISA calculated by regarding $82 \%$ of treated leprosy patients (D) as positive

\begin{tabular}{lccc}
\hline $\begin{array}{l}\text { Subject } \\
\text { group }\end{array}$ & $\begin{array}{c}\text { Bangladesh } \\
\% \text { positive }\end{array}$ & $\begin{array}{c}\text { Fiji } \\
\% \text { positive }\end{array}$ & $\begin{array}{c}\text { Total } \\
\text { positive }\end{array}$ \\
\hline A & $64 \cdot 0(48 / 75)$ & $41 \cdot 7(10 / 24)$ & $58 \cdot 6$ \\
B & $90 \cdot 0(18 / 20)$ & $87 \cdot 0(20 / 23)$ & $88 \cdot 4$ \\
C & $62 \cdot 7(32 / 51)$ & None tested & $62 \cdot 7$ \\
D & $82 \cdot 7(43 / 52)$ & $82 \cdot 1(46 / 56)$ & $82 \cdot 4$ \\
E & $78 \cdot 7(37 / 47)$ & $69 \cdot 2(27 / 39)$ & $74 \cdot 4$ \\
F & None tested & $71 \cdot 4(15 / 21)$ & $71 \cdot 4$ \\
Missing values: & 8 & 0 & 8 \\
\hline All & $72 \cdot 7(178 / 245)$ & $72 \cdot 4(118 / 163)$ & $72 \cdot 5$ \\
\hline
\end{tabular}

A, household contacts; B, hospital contacts; C, untreated patients; D, treated patients; E, Indigenous subjects; F, treated TB patients.

response to $M$. tuberculosis amongst the untreated patients compared with the treated patients $(P<0 \cdot 001)$.

Salivary antibody against the BSA glycoconjugate ${ }^{17}$ which mimics an epitope of PGL1 was only detected in 3 lepromatous patients, all of whom exhibited high absorbance levels.

\section{Discussion}

Both of the ELISA methods used in this study detect antibody against surface antigens of $M$. leprae. Although the whole mycobacterial assay is relatively crude compared with the specific assay developed by Brett et al., ${ }^{17}$ it has proved sufficiently robust to give reproducible results under field conditions and it has the advantage of detecting antibody directed against any external antigens, whether these are specific to $M$. leprae or not. ABTS is a peroxidase substrate which has optimal activity at $25^{\circ} \mathrm{C}^{18}$ and its use in the final stage of the ELISA has proved eminently suitable for assays under field conditions. In Fiji, better results were obtained by performing the HRP-ABTS incubation at $24^{\circ} \mathrm{C}$ than at $32^{\circ} \mathrm{C}$.

The proportion of saliva samples with positive ELISA results for $\operatorname{IgA}$ against $M$. leprae in household contacts of both Fijian and Bangladeshi patients is significantly lower than that found in hospital workers who have a similar (and possibly even greater or longer) exposure to $M$. leprae. Indigenous subjects, with a lower degree of exposure to leprosy bacilli have an intermediate number of positive responses. The increased proportion of treated patients with demonstrable IgA responses against $M$. leprae compared with untreated leprosy patients appears to parallel increases in CMI against $M$. leprae which may occur as a result of treatment ${ }^{14}$ and confirms the previous observation made by Abe et al. ${ }^{11}$

Thus there appears to be a lack of a salivary IgA response to $M$. leprae amongst many apparently healthy contacts of untreated leprosy patients and amongst the patients themselves. Mucosal antibody responses to $M$. tuberculosis and $M$. scrofulaceum are also weaker in these individuals, suggesting that there may be some general suppression of mucosal immunity in both groups affecting mucosal responses to mycobacteria. Various factors have been found to abrogate mucosal immune responses, notably malnutrition and intercurrent infection..$^{8,19}$

Very few salivary antibody responses were detected against the BSA glycoconjugate which mimics an epitope of PGL1, a finding consistent with the low number of positive results obtained in saliva by Abe et al. ${ }^{12}$ using purified PGLl in an ELISA for salivary antibody. This suggests that $\mathrm{PGL} 1$ is not important as an antigen in the mucosal immune response to M. leprae. Comparison of 

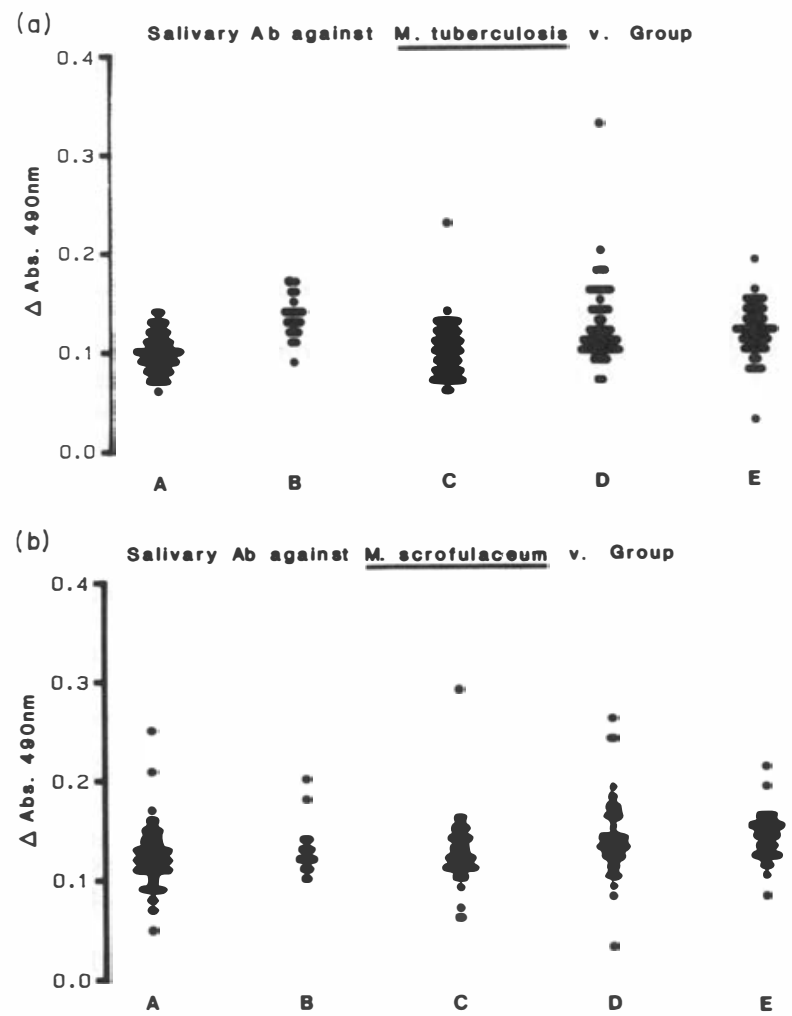

Figure 2. Salivary antibody response to whole y-irradiated: (a) M. tuberculosis $\mathrm{H} 37 \mathrm{Rv}$; and (b) M. scrofulaceum. A, household contacts; B, hospital contacts; C, untreated patients; D, treated patients and; E, indigenous subjects.

the results of salivary and serum ELISAs for antibody against $M$. leprae in the Bangladeshi patients $^{20,21}$ showed little correlation, suggesting that the basis for these responses may be substantially different.

The association between lower levels of secretory IgA against $M$. leprae in household contacts of leprosy patients and their greater risk of developing leprosy, together with the apparent rise in salivary IgA levels following chemotherapy in leprosy patients, leads us to suggest that a mucosal immune response to $M$. leprae may be one of the major protective factors preventing leprosy in those who come into contact with the disease.

Further studies will be required to determine the specificity and possible significance of the $\operatorname{IgA}$ response to $M$. leprae, although an $M$. leprae-specific response may not be essential for protection. We hope to investigate which antigens are involved in the $\operatorname{IgA}$ response against $M$. leprae which should make it possible to produce more sensitive ELISAs for the detection of mucosal immune responses to $M$. leprae. It will be necessary to follow up a large population of leprosy contacts over several years to determine whether a positive IgA anti- $M$. leprae antibody response correlates with protection.

\section{Acknowledgments}

We wish to thank Dr M S Islam, Dr T Ali, Mr S Halda, Dr J Ivanyi, Dr R J W Rees, Professor C du Florey, Dr E C Dawlako, Professor H Lander, and Dr T E Isles for their assistance. The visit by IAC to Bangladesh was supported by the award of the Becton-Dickinson Travelling Scholarship by the 
Royal College of Pathologists and the laboratory work was funded by LEPRA. The visit by NDCS to Fiji was funded by the St Francis Leprosy Guild, Roussel Laboratories Ltd, and LEPRA. We are grateful to Mr R Fawkes and Mr S McPherson for preparation of the figures.

\section{References}

${ }^{1}$ Green CA, Katoch VM, Desikan KV. Quantitative estimation of Mycobacterium leprae in exhaled nasal breath. Lepr Rev, 1983; 54: 337-40.

${ }^{2}$ Pallen MJ, McDermott RD. How might Mycobacterium leprae enter the body? Lepr Rev, 1986; 57: $289-98$.

${ }^{3}$ Bjune G, Closs O, Barnetson RStC. Early events in the host-parasite relationship and immune response in clinical leprosy: its possible importance for leprosy control. Clin exp Immunol, 1983; 54: 289-97.

${ }^{4}$ Godal T, Negassi K. Subclinical inf ection in leprosy. Br Med J, 1973; 3: 557-9.

${ }_{5}^{5}$ Dudley JP. Adherence of microorganisms in infections of the respiratory tract. Laryngoscope, 1982; 92: 68-9.

${ }^{6}$ Neumann AW, Absolom DR, Francis DW, Zingg W, van Oss CJ. Surface thermodynamics of phagocytic ingestion of non-opsonised bacteria by granulocytes in liquids of different surface tensions. Cell Biophys, 1982; 4: 285-93.

${ }^{7}$ Gibbons RJ, Etherden I. Comparative hydrophobicities of oral bacteria and their adherence to salivary pellicles. Infect Immun, 1983; 41: 1190-6.

8 Allardyce RA, Bienenstock J. The mucosal immune system in health and disease with an emphasis on parasitic infection. Bull WHO, 1984; 62: 7-25.

9 Franek J, Libich J, Kubin V. Mechanisms of antibacterial immunity of mucous membranes. Folia Microbiol, 1984; 29: 375-84.

10 Abe M, Minagawa F, Yoshino Y, Itoh M. Immunoglobulin levels and antibody titers in nasal secretions versus serum of leprosy patient. Int J Lepr, 1974; 42: 508 (Abstract).

11 Abe M, Yoshino Y, Minagawa F, Miyaji I, Sampoonachot P, Ozawa T, Sakamoto Y, Saito T, Saikawa K. Salivary immunoglobulins and antibody activities in leprosy. Int J Lepr, 1984; 52: 343-50.

12 Abe M, Mijaji I, Okushita T, Mingawa F, Yoshino Y, Sakamoto Y, Saikawa K. Anti-mycobacterial antibodies in saliva. Lepr Rev, 1986; 57: (Suppl 2), 213-23.

13 Arny M, Kelly-Hatfield P, Lamm ME, Phillips-Quagliata JM. T-cell help for the IgA response: the function of $\mathrm{T}$ cells from different lymphoid organs in regulating the proportions of plasma cells expressing various isotypes. Cell Immunol, 1984; 89: 95-112.

14 Cree IA, Smith WCS, Rees RJW, Beck JS. The influence of anti-mycobacterial chemotherapy on delayed hypersensitivity skin-test reactions in leprosy patients. Lepr Rev, 1988; 59: 145-51.

${ }^{15}$ Ridley DS, Jopling WH. Classification of leprosy according to immunity. A five group system. Int J Lepr, 1966; 34: 255-73.

16 Ridley DS. Histological classification and the immunological spectrum of leprosy. Bull WHO, 1974; 51: 45165.

17 Brett SJ, Payne SN, Gigg J, Burgess P, Gigg R. Use of synthetic glycoconjugates containing the Mycobacterium leprae specific and immunodominant epitope of phenolic glycolipid I in the serology of leprosy. Clin exp Immunol, 1986; 64: 476-83.

18 Porstmann B, Porstmann T, Nugel E, Evers U. Which of the commonly used marker enzymes gives the best results in colorimetric and fluorimetric enzyme immunoassays: horseradish peroxidase, alkaline phosphatase or B-galactosidase? J Immunol Methods, 1985; 79: 27-35.

19 Chandra RK. Nutrition, immunity, and infection: present knowledge and future directions. Lancet, 1983: 1: 688-91.

20 Cree IA. A quantitative study of the relationship between local and systemic immunological parameters in leprosy. PhD Thesis, University of Dundee, 1987, Chapters 8 \& 9 .

${ }^{21}$ Cree IA, Smith WCS, Beck JS. Serum antibody responses to mycobacteria in leprosy patients and their contacts. Lepr Rev, 1988: 59: 317-327. 